\title{
FITOQUÍMICA E QUIMIOSSISTEMÁTICA DE Euxylophora paraensis (Rutaceae)\#
}

Marsele Machado Isidoro, Maria Fátima das Graças Fernandes da Silva*, João Batista Fernandes e Paulo Cezar Vieira Departamento de Química, Universidade Federal de São Carlos, CP 676, 13565-905 São Carlos - SP, Brasil

Alberto C. Arruda e Sebastião da Cruz Silva

Departamento de Química, Universidade Federal do Pará, 66075-900 Belém - PA, Brasil

Recebido em 27/4/12; aceito em 28/8/12; publicado na web em 28/9/12

PHYTOCHEMICAL AND CHEMOSYSTEMATIC STUDIES OF Euxylophora paraensis (Rutaceae). Phytochemical studies of the leaves and stem have led to the identification of the known coumarins isooxypeucedanin, oxypeucedanin hydrate, xanthotoxin, isopimpinellin, 8-methoxymarmesin and marmesin, flavonoids quercetin-3-O- $\alpha$-L-rhamnopyranoside, myricetin-3-O- $\alpha$-Lrhamnopyranoside and hesperidin, alkaloids skimmianine and $\mathrm{N}$-methylflindersine and limonoid limonin. The compounds isolated and the chemical profile of Euxylophora obtained from the literature clearly indicate its phytochemical affinities with other Rutoideae species.

Keywords: Euxylophora paraensis; Rutaceae; chemosystematics.

\section{INTRODUÇÃO}

A família Rutaceae constitui o maior grupo de plantas da ordem Sapindales, possuindo cerca de 160 gêneros, com 1900 espécies amplamente distribuídas nas regiões tropicais e temperadas do globo terrestre, sendo mais abundante na América tropical, sul da África e Austrália. ${ }^{1,2}$ No Brasil existem cerca de 200 espécies já descritas. ${ }^{2}$ As plantas desta família são muito conhecidas pela presença de uma ampla diversidade de metabólitos secundários, destacando-se os alcaloides, especialmente aqueles derivados do ácido antranílico, as cumarinas, as lignanas, os flavonoides e os limonoides. ${ }^{1,3,4}$ Existem relatos de largo espectro de atividades biológicas associadas a estas classes de metabólitos. ${ }^{1,3}$

O gênero Euxylophora é monotípico, apresentando somente a espécie Euxylophora paraensis de ocorrência no norte do Brasil. ${ }^{5}$ Ela é conhecida popularmente como pau-amarelo devido à cor de sua madeira, a qual é de excelente qualidade, encontrando aplicação na confecção de diversos móveis. Suas árvores de grande porte constam na lista de espécies da flora brasileira ameaçadas de extinção desde 1993. ${ }^{6}$ Segundo a literatura, foram isolados de E. paraensis somente alcaloides do tipo $2(1 H)$-quinolinona, $4(1 H)$-quinolinona, ${ }^{7-9}$ indolopiridoquinazolínico e bis-2(1H)-quinolinona. ${ }^{8-16}$ Estas substâncias resultaram do estudo químico da casca do caule e do cerne, não havendo, até o momento, citação sobre fitoquímica das folhas de $E$. paraensis. As cumarinas são de ocorrência ampla nos gêneros de Rutaceae, ou seja, são marcadores químicos da família. A ausência de citações destes compostos para este gênero despertou o interesse no estudo de outros órgãos na tentativa de se buscar as cumarinas. A análise preliminar via cromatografia liquida de alta eficiência (CLAE-UV) de extratos das folhas de E. paraensis indicou a presença das cumarinas, o que levou ao presente estudo. Assim, este trabalho relata o primeiro estudo químico das folhas de E. paraensis que levou ao isolamento das cumarinas iso-oxipeucedanina, oxipeucedanina hidratada, xantotoxina, isopimpenilina, dos flavonoides quercetina-3O- $\alpha$-L-ramnopiranosídeo, miricetina-3-O- $\alpha$-L-ramnopiranosídeo e hesperidina e do alcaloide esquimmianina. $\mathrm{O}$ caule foi reestudado, mas foram analisadas somente as frações que em análise de $\mathrm{RMN}{ }^{1} \mathrm{H}$ mostraram a presença de substâncias não isoladas anteriormente. Duas

*e-mail: dmfs@ufscar.br

\#Artigo em homenagem ao Prof. Otto R. Gottlieb (31/8/1920-19/6/2011) cumarinas 8-metóxi-marmesina e marmesina, o limonoide limonina e o alcaloide $\mathrm{N}$-metilflindersina foram isolados do caule. Exceto $\mathrm{N}$-metilflindersina, as demais substâncias estão sendo relatadas pela primeira vez no gênero.

\section{PARTE EXPERIMENTAL}

\section{Procedimentos experimentais gerais}

Os espectros de RMN ${ }^{1} \mathrm{H}$ e ${ }^{13} \mathrm{C}$ (uni e bidimensionais) foram obtidos em espectrômetro Bruker DRX $400 \mathrm{MHz}$, utilizando-se $\mathrm{CDCl}_{3}$, $\mathrm{CD}_{3} \mathrm{OD}$ e DMSO como solventes e TMS como padrão interno. As separações cromatográficas foram realizadas em colunas utilizando-se gel de sílica 60, 70-230, 230-400 mesh e Sephadex LH-20. As separações por cromatografia líquida de alta eficiência (CLAE) foram realizadas em coluna semipreparativa C-18 Phonomenex ${ }^{\circledR}$ Luna ( $\left(\mathrm{x} \mathrm{d} \mathrm{d}_{\mathrm{c}}=250 \times 4,6 \mathrm{~mm} ; 10 \mu \mathrm{m}\right.$ ), utilizando equipamento Shimadzu LC-8A com válvula de reciclo, injetor Rheodyne 7123 com loop de $500 \mu \mathrm{L}$ e detector UV/VIS Shimadzu SPD-6AV. Os fracionamentos por cromatografia líquida de média pressão (CLMP) foram realizados em equipamento Amersham bioscience P-900 equipado com bomba binária, loop de $500 \mu \mathrm{L}$, coletor automático de $7 \mathrm{~mL}$ por fração com fluxo de $10 \mathrm{~mL} \mathrm{~min}^{-1} \mathrm{e}$ detector UV/VIS. As análises cromatográficas em camada fina foram realizadas em cromatoplacas de sílica gel F-254 sobre placa de alumínio Merck, de 0,2 mm de espessura, empregando-se como revelador a solução ácida de vanilina (5 g de vanilina em $9 \mathrm{~mL}$ de metanol, $0.5 \mathrm{~mL}$ de $\mathrm{H}_{2} \mathrm{SO}_{4}$ e 3 gotas de ácido acético).

\section{Material vegetal}

As folhas de E. paraensis foram coletadas e identificadas pelo Prof. Dr. A. C. Arruda, do Departamento de Química da Universidade Federal do Pará, no dia 05/04/2005 na Fazenda Paulo Maria no Município de Marituba (PA). A exsicata do espécime coletado encontra-se depositada no herbário da EMBRAPA Amazônia Oriental, sob o número 181030.

\section{Preparação dos extratos}

As folhas $(1,3 \mathrm{~kg})$ e caule $(1,0 \mathrm{~kg})$ de E. paraensis foram submetidas à secagem em estufa de ar circulante a $40{ }^{\circ} \mathrm{C}$, por 
aproximadamente $48 \mathrm{~h}$ e pulverizadas em moinho; a extração foi feita com etanol em temperatura ambiente e em repouso, durante 5 dias. Após este período, o solvente foi evaporado em evaporadores rotativos, obtendo-se o extrato bruto.

\section{Isolamento das substâncias de $E$. paraensis}

O extrato etanólico das folhas foi fracionado através de uma partição líquido-líquido gerando 4 extratos: hexânico, diclorometânico, acetato de etila e metanol. Para o estudo do extrato hexânico foi utilizada cromatografia em coluna $\left(\mathrm{h} \mathrm{x} \mathrm{d}_{\mathrm{c}}=45,0 \times 4,0 \mathrm{~cm}\right.$ ) de gel de sílica (230-400 mesh) e eluída em gradiente com hexano, hexano/AcOEt 9:1, hexano/AcOEt 1:1, AcOEt e MeOH em eluição gradiente, fornecendo 42 subfrações. As subfrações de 1-10 continham em mistura os esteroides sitosterol, estigmasterol e campesterol, os quais foram identificados por CG-EM. Das subfrações agrupadas 11-24 (128 mg) foi isolada a substância $\mathbf{3}(11,1 \mathrm{mg})$ através de refracionamento em Sephadex LH-20 com eluição em $\mathrm{CH}_{2} \mathrm{Cl}_{2}: \mathrm{MeOH}$ (1:1). As demais frações foram analisadas por RMN ${ }^{1} \mathrm{H}(200 \mathrm{MHz})$ e indicaram a presença de hidrocarbonetos e ácidos graxos, os quais não eram de interesse quimiossistemático, com isto não foram estudadas.

$\mathrm{O}$ extrato diclorometânico das folhas foi submetido à cromatografia em coluna de gel de sílica (70-230 mesh) e uma pequena camada de florisil $\left(\mathrm{h} \times \mathrm{d}_{\mathrm{c}}=60,0 \times 5,0 \mathrm{~cm}\right.$ ), utilizando-se como eluente hexano/diclorometano/acetona/metanol, levando a 13 frações obtidas após reunião daquelas que mostraram $\mathrm{R}_{\mathrm{f}}$ similares em cromatoplacas de sílica gel (CCD). A análise destas por $\mathrm{RMN}{ }^{1} \mathrm{H}$ (200 MHz) mostrou que somente em 3 delas havia substâncias de interesse quimiossistemático, sendo estas definidas como frações 1-3. A fração 1 foi submetida à cromatografia líquida de média pressão (CLMP) utilizando coluna preparativa Gemini C-18 ( $\mathrm{h} \mathrm{x} \mathrm{d}_{\mathrm{c}}=250 \mathrm{x}$ $21 \mathrm{~mm} ; 10 \mu \mathrm{m})$. Após eluição gradiente com $\mathrm{H}_{2} \mathrm{O} / \mathrm{CH}_{3} \mathrm{CN}(5-100 \%$ B), 56 subfrações de $7 \mathrm{~mL}$ foram coletadas e reunidas após análise por CCD. Esse procedimento resultou no isolamento das substâncias 1 (21 mg) e 2 (11,2 mg), coletadas nos frascos 18 e 21, respectivamente.

$\mathrm{O}$ refracionamento da fração 2 foi feito em coluna Sephadex LH-20 eluída com $\mathrm{MeOH} / \mathrm{CH}_{2} \mathrm{Cl}_{2}$ 7:3, levando a outras subfrações. As subfrações 11 e 12 continham o composto 8 puro, e as subfrações 16-19 foram reunidas em uma única fração que foi reanalisada posteriormente via CLAE semipreparativa. Foi utilizada uma coluna C-18 Phonomenex ${ }^{\circledR}$ Luna ( $\left(\mathrm{x} \mathrm{d}_{\mathrm{c}}=250 \times 4,6 \mathrm{~mm} ; 10 \mu \mathrm{m}\right)$ e eluição com $\mathrm{MeOH} / \mathrm{CH}_{2} \mathrm{Cl}_{2}$ (7:3), fornecendo a substância 4 (5,9 mg).

$\mathrm{O}$ extrato hidroalcoólico foi submetido à cromatografia em coluna de vidro ( $\mathrm{h} \mathrm{x} \mathrm{d}_{\mathrm{c}}=50,0 \times 4,5 \mathrm{~cm}$ ), tendo como fase estacionária Sephadex LH-20 e eluição isocrática (MeOH 100\%). Com a reunião das frações semelhantes foram obtidas 16 , sendo que a fração 7 continha o composto $7(43,4 \mathrm{mg})$ puro. O refracionamento de uma segunda fração através de CLMP, utilizando coluna preparativa Gemini C-18 ( $\left(\mathrm{x} \mathrm{d}_{\mathrm{c}}=250 \times 21 \mathrm{~mm} ; 10 \mu \mathrm{m}\right.$ ) e eluição gradiente com $\mathrm{H}_{2} \mathrm{O} / \mathrm{MeOH}$ $(5-100 \%$ B) levou a 51 subfrações de $7 \mathrm{~mL}$, onde as subfrações 16 e 17 corresponderam aos compostos 5 (10,3 mg) e $\mathbf{6}(12,6 \mathrm{mg})$, respectivamente. As demais frações foram analisadas por $\mathrm{RMN}{ }^{1} \mathrm{H}$ (200 MHz), o que indicou a presença de carboidratos, os quais não eram de interesse quimiossistemático e, por isto, não foram estudadas.

$\mathrm{O}$ extrato etanólico $(2,7 \mathrm{~g})$ do caule foi submetido à cromatografia em coluna de vidro $\left(\mathrm{h} \times \mathrm{d}_{\mathrm{c}}=50,0 \times 4,5 \mathrm{~cm}\right)$, tendo como fase estacionária Sephadex LH-20 e eluição isocrática (MeOH 100\%). Com a reunião das frações semelhantes, foram obtidas 18 frações. A análise destas por $\mathrm{RMN}^{1} \mathrm{H}(200 \mathrm{MHz})$ mostrou que somente em 3 delas havia substâncias de interesse quimiossistemático, sendo estas definidas como frações 1-3. A fração 1 foi submetida à cromatografia em coluna de vidro ( $\mathrm{h} \mathrm{x} \mathrm{d}_{\mathrm{c}}=25,0 \times 3,5 \mathrm{~cm}$ ), tendo como fase estacionária gel de sílica (230-400 mesh) e usando hexano/diclorometano/ acetona/metanol em eluição gradiente, chegou-se a 6 subfrações obtidas após reunião daquelas que mostraram $\mathrm{R}_{\mathrm{f}}$ similares em CCD. $\mathrm{O}$ refracionamento da subfração 5 em cromatografia em camada delgada preparativa em placa de vidro (sílica gel, $\mathrm{h}=25,0$ × 25,0 cm), eluída com acetona/hexano 4:6 forneceu os compostos $9(1,6 \mathrm{mg}) \mathrm{e}$ $10(2,2 \mathrm{mg})$. A fração 2 também foi submetida à cromatografia sob as mesmas condições da fração 1 , obtendo-se uma subfração a qual foi recromatografada, utilizando-se Sephadex LH-20 em coluna de vidro $(\mathrm{h} \mathrm{x} \mathrm{d}=25,0 \times 3,5 \mathrm{~cm})$, fornecendo 6 subfrações obtidas após reunião daquelas que mostraram $R_{f}$ similares em CCD. Somente a subfração 5 foi reestudada por CLAE semipreparativa, utilizando uma coluna C-18 Phenomenex ${ }^{\circledR}$ Luna $\left(\mathrm{h} \mathrm{x} \mathrm{d}_{\mathrm{c}}=250 \mathrm{x}\right.$ 4,6 mm; 10 $\mu \mathrm{m})$ e eluição com $\mathrm{MeOH} / \mathrm{CH}_{2} \mathrm{Cl}_{2}$ (7:3), fornecendo a substância $11(50,1 \mathrm{mg})$. A fração 3 foi submetida à cromatografia líquida de média pressão (CLMP), utilizando coluna preparativa Gemini C-18 (h x d $=250 \times 21 \mathrm{~mm} ; 10 \mu \mathrm{m})$, gradiente $\mathrm{H}_{2} \mathrm{O} / \mathrm{CH}_{3} \mathrm{CN}(5-100 \%$ B), detector UV (365, 254 e $217 \mathrm{~nm})$, loop de 2,5 mL e vazão 10,0 $\mathrm{mL} / \mathrm{min}$. Após a eluição foram obtidas 34 subfrações de $14 \mathrm{~mL}$ e a subfração 14 continha 20,6 mg da substância $\mathbf{1 2}$. As demais frações não foram estudas, pois não continham substâncias de interesse quimiossistemático.

\section{Xantotoxina (1)}

Sólido branco. RMN ${ }^{1} \mathrm{H}\left(400 \mathrm{MHz}, \mathrm{CDCl}_{3}\right), \delta_{\mathrm{H}}$ (mult., $J$ em Hz, H): 7,75 ( $d$, 9,5, H-4), 7,68 (d, 2,5, H-2'), 7,34 ( $s, \mathrm{H}-5), 6,80$ (d, 2,5, H-3') , 6,35 (d, 9,5, H-3), 4,28 ( $\left.s, 8-\mathrm{OCH}_{3}\right)$. RMN ${ }^{13} \mathrm{C}(50 \mathrm{MHz}$, $\left.\mathrm{CDCl}_{3}\right) \delta_{\mathrm{C}}: 160,3(\mathrm{C}-2), 147,8(\mathrm{C}-7), 146,6(\mathrm{C}-2$ '), 144,2 (C-4), 146,6 (C-2'), 143,1 (C-8a), 132,9 (C-8), 126,1 (C-6), 116,5 (C-4a), 114,8 (C-3), 112,9 (C-5), 61,3 (8- $\left.\mathrm{OCH}_{3}\right)$.

\section{Isopimpenilina (2)}

Sólido branco. RMN ${ }^{1} \mathrm{H}\left(400 \mathrm{MHz}, \mathrm{CDCl}_{3}\right), \delta_{\mathrm{H}}$ (mult., $J$ em Hz, H): 8,11 (d, 9,3, H-4), 7,61 (d, 2,5, H-2'), 6,99 (d, 2,5, H-5), 6,28 (d, 9,3, H-3'), 4,16 (s, 5- $\left.\mathrm{OCH}_{3}\right), 4,16\left(s, 8-\mathrm{OCH}_{3}\right)$. RMN ${ }^{13} \mathrm{C}(50 \mathrm{MHz}$, $\left.\mathrm{CDCl}_{3}\right) \delta_{\mathrm{C}}: 160,5$ (C-2), 150,0 (C-7), 145,2 (C-2'), 144,3 (C-5), 145,2 (C-2'), 143,7 (C-8a), 139,4 (C-4), 128,3 (C-8), 114,8 (C-6), 107,7 (C-4a), 112,9 (C-3), 105,1 (C-3'), 60,8 (8- $\left.\mathrm{OCH}_{3}\right), 61,7\left(5-\mathrm{OCH}_{3}\right)$.

\section{Iso-oxipeucedanina (3)}

Sólido branco. RMN ${ }^{1} \mathrm{H}\left(400 \mathrm{MHz}, \mathrm{CDCl}_{3}\right), \delta_{\mathrm{H}}$ (mult., $J$ em Hz, $\mathrm{H}): 8,33$ ( $d d, 9,6$ e 0,6, H-4), 7,61 (d, 2,4, H-2'), 7,21 (dd, 0,8 e 0,6, H-8), 6,84 (dd, 2,4 e 0,8, H-3'), 6,35 (d, 9,6, H-3), 5,08 ( $s, \mathrm{H}-1$ '”), 2,87 ( $m, \mathrm{H}-3$ ") $, 1,19$ (d, 6,8, H-4"), 1,19 (d, 6,8, H-5"). RMN ${ }^{13} \mathrm{C}$ (50 MHz, $\left.\mathrm{CDCl}_{3}\right) \delta_{\mathrm{C}}: 161,0$ (C-2), 158,0 (C-7), 145,5 (C-2'), 147,0, (C-5), 145,5 (C-2'), 152,0 (C-8a), 139,1 (C-4), 95,1 (C-8), 114,0 (C-6), 108,0 (C-4a), 113,3 (C-3), 104,1 (C-3'), 75,0 (C-1'”), 209,0 (C-2”), 37,4 (C-3”), 17,9 (C-4”), 29,5 (C-5”).

\section{Oxipeucedanina hidratada (4)}

Sólido branco. RMN ${ }^{1} \mathrm{H}\left(400 \mathrm{MHz}, \mathrm{CDCl}_{3}\right), \delta_{\mathrm{H}}$ (mult., $J$ em $\mathrm{Hz}, \mathrm{H}): 8,18$ (dd, 9,6 e 0,8, H-4), 7,61 (d, 2,5, H-2'), 7,19 (dd, 0,8 e $0,6, \mathrm{H}-8), 6,99$ ( $d d, 2,5$ e $0,6, \mathrm{H}-3$ '), 6,31 (d, 9,5, H-3), 4,45 (dd, 10,0 e 3,0 2"'a), 4,45 ( $d d, 10,0$ e 3,0 2"'b), 3,91 ( $m, \mathrm{H}-3$ ") ), 2,81 ( $s l$, OH-3"), 1,31 ( $s, \mathrm{H}-5$ "), ( $s, \mathrm{H}-6 ")$. RMN ${ }^{13} \mathrm{C}\left(50 \mathrm{MHz}, \mathrm{CDCl}_{3}\right) \delta_{\mathrm{C}}$ : 160,8 (C-2), 157,7 (C-7), 145,5 (C-2'), 148,9 (C-5), 145,5 (C-2'), 153,7 (C-8a), 139,1 (C-4), 95,1 (C-8), 113,5 (C-6), 107,8 (C-4a), 113,3 (C-3), 104,1 (C-3'), 74,8 (C-2”), 72,1 (C-3”), 76,9 (C-4”), 24,6 (C-5"), 26,2 (C-6”).

\section{Quercetina-3-O- $\alpha$-L-ramnopiranosídeo (5)}

Sólido amarelo. RMN ${ }^{1} \mathrm{H}\left(400 \mathrm{MHz}, \mathrm{CD}_{3} \mathrm{OD}\right), \delta_{\mathrm{H}}$ (mult., $J$ em Hz, H): 7,34 (d; H-2'), 7,31 (dd, 8,0 e 2,0, H-6'), 6,91 (d, 8,0, H-5'), 6,37 (d, 2,0, H-8), 6,20 (d, H-6), 5,34 (d, 1,8, H-1"), 4,22 (dd, 3,2 e 
1,8, H-2"), 3,74 (dd, 9,4 e 3,2, H-3"), 3,41 ( $m, \mathrm{H}-5$ "), 3,33 ( $m, \mathrm{H}-4 ")$, $0,94(d, 5,6, \mathrm{H}-6 ")$. RMN ${ }^{13} \mathrm{C}\left(50 \mathrm{MHz}, \mathrm{CD}_{3} \mathrm{OD}\right) \delta_{\mathrm{C}}: 179,5(\mathrm{C}-4)$, 165,8 (C-7), 163,2 (C-5), 158,9 (C-2), 159,0 (C-9), 149,8 (C-4'), 146,2 (C-3'), 136,0 (C-3), 122,1 (C-1'), 123,0 (C-6'), 116,5 (C-2'), 116,4 (C-5'), 105,9 (C-10), 103,5 (C-1"), 99,8 (C-6), 94,7 (C-8), 73,0 (C-4"), 72,2 (C-3"), 71,2 (C-2”), 71,8 (C-5"), 17,9 (C-6").

\section{Miricetina-3-O- $\alpha$-L-ramnopiranosídeo (6)}

RMN ${ }^{1} \mathrm{H}\left(\mathrm{CD}_{3} \mathrm{OD}\right), \delta_{\mathrm{H}}$ (mult., $J$ em Hz, H): $\delta$ 0,94 (3H, $d, 5,6$, H-6"), 3,33 ( $t, 9,2, \mathrm{H}-4$ "), 3,51 ( $m, \mathrm{H}-5$ "), 3,78 ( $d d, 9,2$ e 3,2, H-3"), 4,21 (dd,3,2 e 1,8, H-2"'), 5,31 (d, 2,0, H-1 ") ), 6,20 (d, 2,0, H-6), 6,42 (d, 2,0, H-8), 6,95 (2H, s, H-2', H-6' ); RMN ${ }^{13} \mathrm{C}\left(50 \mathrm{MHz}, \mathrm{CD}_{3} \mathrm{OD}\right)$ $\delta_{\mathrm{C}}: 17,8(\mathrm{C}-6 "), 71,8(\mathrm{C}-2 "), 72,0(\mathrm{C}-5 "), 72,2$ (C-3"), 72,3 (C-4"), 94,9 (C-8), 100,0 (C-6), 103,8 (C-1"'), 106,0 (C-10), 108,5 (C-6'), 109,7 (C-2'), 122,1 (C-1'), 136,5 (C-3), 138,1 (C-4'), 147,0 (C-3' ,C-5' ), 158.7 (C-9), 159,6 (C-2), 163,4 (C-5), 166,3 (C-7), 159,6 (C-2), 179,8 (C-4).

\section{Hesperidina (7)}

Sólido amorfo amarelado, RMN ${ }^{1} \mathrm{H}\left(400 \mathrm{MHz}, \mathrm{DMSO}-\mathrm{d}_{6}\right) \delta_{\mathrm{H}}$ (mult., $J$ em Hz, H): 5,38 (dd, 3,0 e 12,6, H-2), 2,72 (dd, 3,0 e 14,0, H-3eq), 3,14 ( $d d, 14,0$ e 12,6, H-3ax), 6,05 ( $s l, \mathrm{H}-6), 6,91$ ( $s l, \mathrm{H}-8)$, 6,95 ( $m$, H-2', H-5', H-6'), 4,50 ( $s, \mathrm{H}-1$ '”'), 4,98 (d, 8,1, H-1"), 3,09-3,90 (H-2"-H-5"'), 0,96 (d, 6,0, H-6"), 12,00 ( $s, 5-\mathrm{OH}), 3,86$ $\left(s, 4^{\prime}-\mathrm{OCH}_{3}\right) . \mathrm{RMN}^{13} \mathrm{C}\left(50 \mathrm{MHz}, \mathrm{DMSO}-\mathrm{d}_{6}\right) \delta_{\mathrm{C}}: 78,8(\mathrm{C}-2), 42,3$ (C-3), 197,4 (C-4), 165,4 (C-5), 96,8 (C-6), 163,3 (C-7), 95,9 (C-8), 162,8 (C-9), 103,6 (C-10), 131,0 (C-1'), 114,2 (C-2'), 146,1 (C-3'), 148,0 (C-4'), 112,0 (C-5'), 118,0 (C-6'), 100,9 (C-1'), 73,3 (C-2”), 76,6 (C-3”), 69,9 (C-4”), 75,7 (C-5”), 66,4 (C-6”), 99,8 (C-1"'), 70,6 (C-2"”), 71,1 (C-3"'), 72,5 (C-4"”), 68,7 (C-5"'), 18,2 (C-6"'), $56,0\left(4^{\prime}-\mathrm{OCH}_{3}\right)$.

\section{Esquimmianina (8)}

$\mathrm{RMN}{ }^{1} \mathrm{H}\left(400 \mathrm{MHz}, \mathrm{CDCl}_{3}\right) \delta_{\mathrm{H}}$ (mult., $J$ em Hz, H): 8,03 (d, 9,4, H-5), 7,24 (d, 9,4, H-6), 7,59 (d, H-2'), 7,05 (d, 2,8, H-3'), 4,44 ( $s$, $\left.4-\mathrm{OCH}_{3}\right), 4,04\left(s, 7-\mathrm{OCH}_{3}\right), 4,12\left(s, 8-\mathrm{OCH}_{3}\right)$.

\section{Marmesina (9)}

$\mathrm{RMN}{ }^{1} \mathrm{H}\left(400 \mathrm{MHz}, \mathrm{CDCl}_{3}\right) \delta_{\mathrm{H}}$ (mult., $J$ em Hz, H): 7,60 (d, 9,4, H-4), 6,22 (d, 9,4, H-3), 6,75 ( $s, \mathrm{H}-8), 7,22$ ( $s l, \mathrm{H}-5), 4,74$ ( $d d, 9,2$ e 8,5, H-2'), 3,22 ( $d d d, 14,8,8,5$ e 1,0, H-3'a), 3,20 ( $d d d, 14,8,9,2$ e 1,0, H-3'b), 1,38 (3H, s, H-2"), 1,24 (3H, s, H-3"). RMN ${ }^{13} \mathrm{C}(50$ $\left.\mathrm{MHz} \mathrm{CDCl}_{3}\right) \delta_{\mathrm{C}}: 161,1$ (C-2), 163,3 (C-7), 91,1 (C-2'), 123,4 (C-5), 29,5 (C-3'), 155,7 (C-8a), 139,4 (C-4), 97,9 (C-8), 125,0 (C-6), 113,7 (C-4a), 112,3 (C-3), 71,6 (C-1"), 26,2 (C-2"), 24,2 (C-3").

\section{8-Metóxi-marmesina $(\mathbf{1 0})$}

$\mathrm{RMN}{ }^{1} \mathrm{H}\left(400 \mathrm{MHz}, \mathrm{CDCl}_{3}\right) \delta_{\mathrm{H}}$ (mult., $J$ em Hz, H): 7,58 (d, 9,5, H-4), 6,22 (d, 9,5, H-3), 6,96 ( $s l, \mathrm{H}-5), 4,78$ ( $d d, 9,2$ e 8,5, H-2') , 3, 25 ( $d d d, 14,8,8,5$ e 1,0, H-3'a), 3,20 ( $d d d, 14,8,9,2$ e 1,0, H-3'b), 1,39 (3H, s, H-2") ), 1,25 (3H, s, H-3"), 4,06 (3H, s, 8- $\left.\mathrm{OCH}_{3}\right) . \mathrm{RMN}{ }^{13} \mathrm{C}$ $\left(50 \mathrm{MHz}, \mathrm{CDCl}_{3}\right) \delta_{\mathrm{C}}: 161,0$ (C-2), 154,4 (C-7), 91,5 (C-2'), 117,2 (C-5), 29,9 (C-3'), 147,5 (C-8a), 143,5 (C-4), 132,0 (C-8), 125,9 (C-6), 113,7 (C-4a), 112,5 (C-3), 71,6 (C-1”), 26,0 (C-2”), 24,4 (C-3"), 61,0 (8- $\left.\mathrm{OCH}_{3}\right)$.

\section{$N$-Metilflindersina (11)}

$\mathrm{RMN}{ }^{1} \mathrm{H}\left(400 \mathrm{MHz}, \mathrm{CDCl}_{3}\right.$ ) $\delta_{\mathrm{H}}$ (mult., $J$ em Hz, H): 7,97 (dd, 8,4 e 2,0, H-5), 7,23 ( $d d d, 8,6,6,8$ e 2,0, H-6), 7,55 ( $d d d, 8,4,6,8$ e 1,6, H-7), 7,32 ( $d l, \mathrm{H}-8$ ), 6,76 ( $d, 9,9, \mathrm{H}-4$ ') , 5,54 ( $d$, 9,9, H-3'), 1,52 $\left(2 \mathrm{H}, s, 2\right.$ ' $\left(\mathrm{CH}_{3}\right), 3,70\left(3 \mathrm{H}, s, \mathrm{~N}-\mathrm{CH}_{3}\right) . \mathrm{RMN}{ }^{13} \mathrm{C}\left(50 \mathrm{MHz}, \mathrm{CDCl}_{3}\right)$ $\delta_{\mathrm{C}}: 161,0(\mathrm{C}-2), 106,2(\mathrm{C}-3), 155,0(\mathrm{C}-4), 116,2$ (C-5a), 123,0 (C-5), 122,0 (C-6), 131,0 (C-7), 114,0 (C-8), 139,5 (C-8a), 117,9 (C-4'),
125,5 (C-3'), 78,8 (C-2'), 29,2 (2'( $\left.\left.\mathrm{CH}_{3}\right)\right), 28,2\left({\left.\mathrm{~N}-\mathrm{CH}_{3}\right)}^{\prime}\right.$.

\section{Limonina (12)}

Sólido branco, RMN ${ }^{1} \mathrm{H}\left(400 \mathrm{MHz}, \mathrm{CDCl}_{3}\right) \delta_{\mathrm{H}}$ (mult., $J$ em Hz, H): $\delta 4,04$ ( $s l, \mathrm{H}-1), 2,68$ ( $d d, 16,8$ e 1,9, H-2a), 2,98 (dd, 16,8 e 3,8, H-2b), 2,23 ( $d d, 15,8$ e 3,2, H-5), 2,86 (dd,15,8 e 14,5, H-6 ax), 2,47 ( $d d, 14,5$ e 3,2 H-6 eq), 2,55 ( $d d, 12,2$ e 2,8, H-9), 1,78 ( $m, \mathrm{H}-11 \mathrm{a})$, 1,90 ( $m, \mathrm{H}-11 \mathrm{~b}), 1,84$ ( $m, \mathrm{H}-12 \mathrm{ax}), 1,51$ ( $m, \mathrm{H}-12 \mathrm{eq}), 4,04$ ( $s, \mathrm{H}-15)$, 5,47 ( $s, \mathrm{H}-17), 1,18$ (3H, $s, \mathrm{H}-18), 4,47$ (d,13,0, H-19a), 4,77 (dd, $13,0$ e $0,6 \mathrm{H}-19 \mathrm{~b}), 7,42$ ( $t l, \mathrm{H}-21), 6,34$ ( $d d, 1,7$ e $0,7, \mathrm{H}-22), 7,40$ ( $t l, \mathrm{H}-23), 1,30$ (3H, $s, \mathrm{H}-28), 1.19$ (3H, s, H-29), 1,30 (3H, s, H-30). $\mathrm{RMN}{ }^{13} \mathrm{C}\left(\mathrm{CDCl}_{3}\right) \delta_{\mathrm{C}}: 79,2$ (C-1), 35,7 (C-2), 169, 1 (C-3), 80,4 (C4), 60,7 (C-5), 36,5 (C-6), 206,1 (C-7), 51,4 (C-8), 48, 2 (C-9), 46,0 (C-10), 19,0 (C-11), 30,9 (C-12), 38,0 (C-13), 65,7 (C-14), 53,9 (C-15), 166,0 (C-16), 77,9 (C-17), 20,8 (C-18), 65,4 (C-19), 120,0 (C-20), 141,2 (C-21), 109,7 (C-22), 143,3 (C-23), 30,2 (C-28), 21,5 (C-29), 17,7 (C-30).

\section{RESULTADOS E DISCUSSÃO}

O estudo fitoquímico dos extratos das folhas de E. paraensis levou ao isolamento de quatro cumarinas (1-4), três flavonoides (5-7) e o alcaloide esquimmianina (8) (Figura 1).

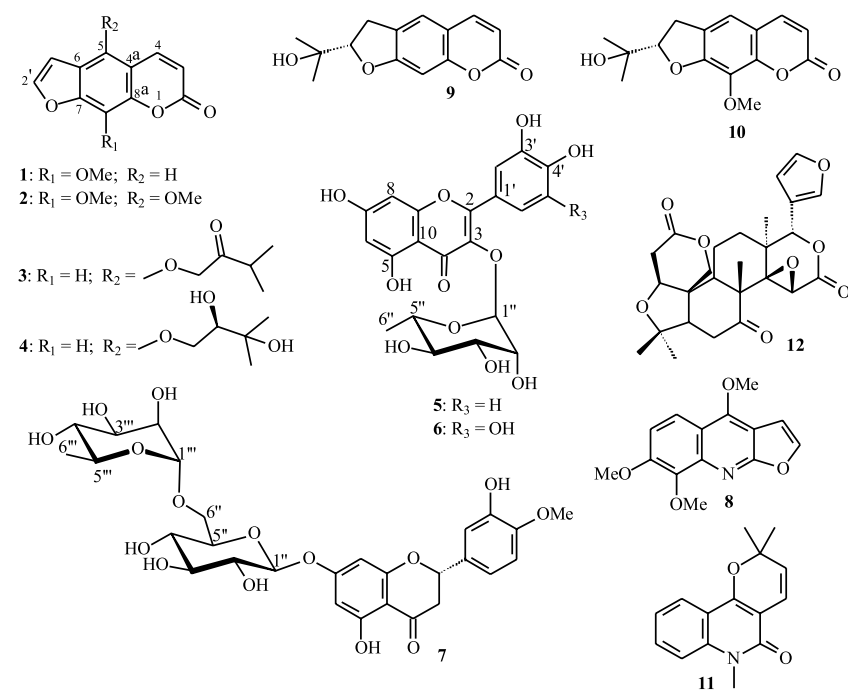

Figura 1. Compostos isolados de E. paraensis

As substâncias 1-4 apresentaram no espectro de RMN ${ }^{1} \mathrm{H}$ (solvente: $\left.\mathrm{CDCl}_{3}\right)$ um par de dubletos $(J \cong 9,5 \mathrm{~Hz})$ localizados entre $\delta$ 6,1-6,4 e 7,5-8,3, sugerindo a presença de um esqueleto cumarínico, sendo estes sinais característicos dos hidrogênios H-3 e H-4, respectivamente. ${ }^{17}$ Também foi observada para estas substâncias a presença de um anel furano $\alpha, \beta$-substituído, sendo facilmente reconhecido através de um par de dubletos $(J=2,5 \mathrm{~Hz})$ em $\delta 7,6$ e 6,8, atribuídos a H-2' e H-3', respectivamente. O sinal deste último hidrogênio é observado como um duplo dubleto $(J=2,4$ e 0,8$)$ quando C-8 se encontra hidrogenado, devido a um acoplamento a longa distância $\left({ }^{5} J\right)$ deste com H-3' e isto foi observado para as substâncias 3 e 4. A substância 1 apresentou, além dos sinais relatados acima, um singleto em $\delta 7,34$ e outro em $\delta 4,28$, sendo este último característico do grupo metoxila. A substância 2 apresentou um singleto em $\delta 4,16(6 \mathrm{H})$ atribuído a duas metoxilas. Os substituintes presentes em C-5 das substâncias 3 e 4 foram determinados através das correlações observadas nos mapas de contorno de HSQC e HMBC, que estão representadas na Figura 2.

Os espectros de $\mathrm{RMN}{ }^{13} \mathrm{C}$ destas cumarinas apresentaram sinais característicos para o esqueleto furanocumarínico: $\delta$ 160,3-161,0 

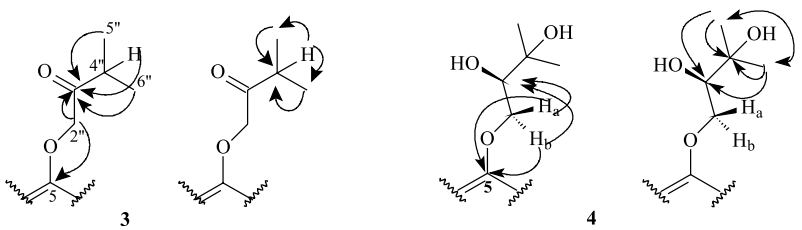

Figura 2. Correlações observadas nos mapas de contorno HMBC para os substituintes presentes em C-5 das substâncias $\mathbf{3}$ e $\mathbf{4}$

$(\mathrm{C}-2) ; \delta 112,9-114,8(\mathrm{C}-3) ; \delta 139,1-144,2$ (C-4); $\delta$ 145,0-146,6 (C-2'); $\delta$ 104,1-106,7 (C-3'). O conjunto de dados acima e a comparação com a literatura permitiram a identificação das cumarinas xantotoxina (1) ${ }^{18}$ isopimpinilina $(\mathbf{2}),{ }^{19}$ iso-oxipeucedanina $(\mathbf{3}) \mathrm{e}$ oxipeucedanina hidratada (4). ${ }^{18,20} \mathrm{~A}$ cumarina $\mathbf{3}$ foi isolada do extrato hexânico e as demais $(\mathbf{1}, \mathbf{2}, \mathbf{4})$ do extrato diclorometânico.

A configuração do carbono quiral da unidade prenila do composto 4 foi considerada $R$ baseando-se no valor de rotação específica obtida $\left([\alpha]_{\mathrm{D}}^{25}=+21\right)$ e comparação deste com aquele citado na literatura para esta mesma substância $\left([\alpha]_{D}{ }^{25}=+8\right)$, a qual além de ser denominada oxipeucedanina hidratada é também conhecida como prangol. ${ }^{21}$ Definir a configuração deste carbono baseando-se na rotação específica obtida foi possível devido à molécula possuir somente este estereocentro.

O estudo do extrato hidroalcoólico forneceu os compostos $\mathbf{5 , 6}$ e 7, sendo este o primeiro relato do isolamento de flavonoides nesta espécie. Os espectros de RMN ${ }^{1} \mathrm{H}$ de 5 e $\mathbf{6}$ apresentaram, na região de hidrogênios aromáticos, um par de dubletos em $\delta$ 6,20 e 6,37 $(J=$ $2,0 \mathrm{~Hz}$ ), característico de sistema de acoplamento $\mathrm{AB}$, correspondente a dois hidrogênios meta-posicionados e atribuídos, respectivamente, a H-6 e H-8 do anel A de um flavonoide. A diferença observada nos espectros destas substâncias está relacionada ao anel B, que apresenta para 5 um sistema de substituição nas posições 3' e 4', sugerido pelos sinais em $\delta 7,34(d, J=2,0 \mathrm{~Hz}), 7,31(d d, J=8,0$ e $2,0 \mathrm{~Hz})$ e $6,91(d, J=8,0 \mathrm{~Hz})$, atribuídos aos hidrogênios H-2', H-6' e H-5', respectivamente; para $\mathbf{6}$ foi observado apenas um singleto em $\delta 6,95$ relativo a dois hidrogênios, característico dos hidrogênios H-2' e H-6' do anel B de uma flavona. A presença de sinais entre $\delta 3,3$ e $\delta 4,2$ nos espectros de $\mathrm{RMN}{ }^{1} \mathrm{H}$ de $\mathbf{5}$ e $\mathbf{6}$ indicou que as duas substâncias se tratavam de flavonoides glicosilados. Esta afirmação pode ser confirmada pelos sinais em $\delta 4,21(d d, J=3,2$ e 1,8 Hz), 3,78 ( $d d, J$ $=9,2$ e $3,2 \mathrm{~Hz}), 3,37(m, 2 \mathrm{H})$ e pelo dubleto em $\delta 0,90(\mathrm{~J}=5,6 \mathrm{~Hz})$, este último sendo característico do grupo metila da ramnose e, assim, o sinal em $\delta 5,50(s l)$ foi atribuído ao hidrogênio anomérico (H-1") da molécula de açúcar. A estereoquímica do carbono anomérico da ramnose e a posição na qual o açúcar está ligado à aglicona foi determinada pela constante de acoplamento de H-1" e por comparação com os dados da literatura, ${ }^{22,23}$ sendo possível identificar a substância quercetina-3-O- $\alpha$-L-ramnopiranosídeo (5) e miricetina-3-O- $\alpha$-Lramnopiranosídeo (6). ${ }^{23}$

O padrão de substituição no anel A do flavonoide 7 foi definido como 5,7-dioxigenado pela presença de dois singletos largos em $\delta$ 6,05 (H-6) e 6,91 (H-8), no espectro de RMN ${ }^{1} \mathrm{H}$, formando um sistema de acoplamento AB. Para o anel B foi observado um multipleto entre 6,75-6,95 atribuído aos hidrogênios H-2', H-5' e H-6', sugerindo anel B 3'-hidróxi-4'-metoxilado ou 4'-hidróxi-3'-metoxilado. $\mathrm{O}$ sinal do grupo metoxila foi observado em $\delta 3,86$ $(3 \mathrm{H})$. Esta substância foi definida como flavanona devido à presença dos sinais de hidrogênios alifáticos $\delta 3,14(d d, J=14,0$ e 12,6 $\mathrm{Hz}), 2,72(d d, J=14,0$ e $3,0 \mathrm{~Hz})$ e $\delta 5,38(d d, J=12,6$ e $3,0 \mathrm{~Hz})$, que foram atribuídos aos hidrogênios $\mathrm{H}-3$ pseudoax, H-3pseudoeq e H-2 do anel C, respectivamente. A presença de sinais na região $\delta$ 3,09-3,86 indicou a presença de unidades de açúcar na estrutura proposta. Esta afirmação pode ser confirmada pelos sinais em $\delta$ $4,98(d, J=8,1 \mathrm{~Hz})$ e 4,50 $(s)$ e pelo dubleto em $\delta 0,96(J=6,0 \mathrm{~Hz}$, $3 \mathrm{H})$, este último sendo característico do grupo metila da ramnose. Estes dados sugeriram a presença do substituinte O- $\beta$-D-rutinosil e permitiram atribuir os sinais em $\delta$ 4,98 e 4,50 aos hidrogênios anoméricos H-1" e H-1". A comparação dos deslocamentos químicos de $\mathrm{RMN}{ }^{1} \mathrm{H} \mathrm{e}{ }^{13} \mathrm{C}$ desta flavanona isolada com os dados descritos na literatura para a hesperidina mostrou que o flavonoide 7 referia-se a esta substância (3',5-di-hidróxi-4'-metoxiflavanona-7-O- $\beta$-[Lramnopiranosil $(\alpha 1 \rightarrow 6)]$-D-glucopiranosídeo, ou hesperetina-7-O$\beta$-[L-ramnopiranosil $(\alpha 1 \rightarrow 6)]$-D-glucopiranosídeo). ${ }^{24}$

Somente um alcaloide foi isolado das folhas e este foi obtido do extrato diclorometânico. Seu espectro de RMN ${ }^{1} \mathrm{H}$ apresentou dois dubletos em $\delta$ 7,59 e 7,05, de hidrogênios furânicos, característicos dos alcaloides furoquinolínicos, e um par de dubletos em $\delta$ 8,03 e 7,24 com $J=9,4 \mathrm{~Hz}$, atribuídos a H-5 e H-6, respectivamente. A presença de três sinais de metoxilas, em $\delta 4,44,4,04$ e 4,12 e a comparação com dados da literatura permitiu identificar esta substância como sendo a esquimmianina $(\mathbf{8}) .^{25}$

O estudo fitoquímico do extrato etanólico do caule de E. paraensis levou ao isolamento de duas cumarinas (9 e 10), do alcaloide $\mathrm{N}$-metilflindersina (11) e do limonoide limonina (12) (Figura 1). As cumarinas 9 e $\mathbf{1 0}$ apresentam em seus espectros de RMN ${ }^{1} \mathrm{H}$ os sinais característicos do esqueleto cumarínico com a presença do anel di-hidrofurano, o qual foi evidenciado pelos sinais em $\delta 4,78$ ( $d d, 9,3$ e $8,5 \mathrm{~Hz}, \mathrm{H}-2$ ') $, 3,25$ ( $d d d, 14,8,8,5$ e $1,3 \mathrm{~Hz}, \mathrm{H}-3$ 'a) e 3,20 ( $d d d$, $14,8,9,3$ e $0,9 \mathrm{~Hz}, \mathrm{H}-3$ 'b). Ainda no espectro de $\mathrm{RMN}{ }^{1} \mathrm{H}$ foi possível observar sinais que caracterizam um grupo hidróxi-isopropila. Esses sinais apresentaram-se como singletos em $\delta 1,39(3 \mathrm{H}, s)$ e $1,25(3 \mathrm{H}, s)$. No espectro da cumarina 9 foram observados dois singletos em $\delta 6,75$ $(1 \mathrm{H}, s)$ e 7,22 (1H,sl) atribuídos, respectivamente, aos H-8 e H-5, sugerindo a presença da marmesina. ${ }^{26}$ Para a segunda cumarina foram observados dois singletos, um em $\delta 4,06(3 \mathrm{H}, s)$ relativo a um grupo metoxila posicionado em C-8, e o outro em $\delta 6,96$ atribuído ao H-5, permitindo identificar a 8-metóxi-marmesina (10). ${ }^{26} \mathrm{~A}$ confirmação destas estruturas foi obtida através de comparação de seus dados de $\mathrm{RMN}$ de ${ }^{13} \mathrm{C}$ com aqueles citados na literatura para a marmesina (9) e seu derivado 8-metóxi-marmesina (10). ${ }^{26}$

O espectro de $\mathrm{RMN}{ }^{1} \mathrm{H}$ da substância 11 mostrou sinais característicos de anel aromático orto-dissubstituído, $\delta 7,97(1 \mathrm{H}, J=8,4$ e 2,0 $\mathrm{Hz}), 7,23(1 \mathrm{H}, J=8,4 ; 6,8$ e 1,6 Hz), 7,55 (1H, $J=8,6$; 6,8 e 2,0 Hz), $7,32(1 \mathrm{H}, J=8,6 ; 1,6 \mathrm{~Hz})$, atribuídos aos $\mathrm{H}-5-\mathrm{H}-8$, respectivamente. $\mathrm{O}$ espectro de $\mathrm{RMN}{ }^{13} \mathrm{C}$ mostrou sinal característico de lactama em $\delta 161,0$, indicando a presença de alcaloide $2(1 H)$-quinolin-2-ona. A ausência de sinais para H-3 e H-4 de um alcaloide $2(1 H)$-quinolin2-ona e a presença de sinais típicos do anel 2,2-dimetilcromeno (um singleto integrando para 6 hidrogênios em $\delta 1,52$ referente a duas metilas geminais e dois dubletos em $\delta 5,54$ e $6,76 \mathrm{com} J=10,0 \mathrm{~Hz}$ referente a hidrogênios olefinicos), permitiram identificar este alcaloide como a N-metilflindersina (11). ${ }^{27}$

Os espectros de RMN ${ }^{1} \mathrm{H}$ e ${ }^{13} \mathrm{C}$ da substância 12 mostraram sinais característicos do anel furano $\beta$-substituído, $\delta_{\mathrm{H}} 7,42(J=0,7$ $\mathrm{Hz}, \mathrm{H}-21), 7,40(\mathrm{~J}=1,7 \mathrm{~Hz}, \mathrm{H}-23), 6,34(J=1,7$ e $0,7 \mathrm{~Hz}, \mathrm{H}-22), \delta_{\mathrm{C}}$ 120,0 (C-20), 141,2 (C-21), 109,7 (C-22) e 143,3 (C-23). O espectro de $\mathrm{RMN}{ }^{1} \mathrm{H}$ ainda mostrou quatro singletos intensos com integração para três hidrogênios em $\delta$ 1,18 (Me-18), 1,30 (Me-28), 1,19 (Me-29) e 1,07 (Me-30) referentes a grupos metila ligados a carbono $\mathrm{sp}^{3} \mathrm{e}$ atribuídos com o auxílio dos mapas de correlações HSQC e HMBC. A presença de sinais em $\delta 4,77(d d, J=13,0$ e $0,6 \mathrm{~Hz}), 4,47(d, J=$ $13,0 \mathrm{~Hz})$ e 4,04 ( $s l$, atribuídos aos hidrogênios geminais do C-19 e $\mathrm{H}-1$, respectivamente, sugeriram tratar-se de um limonoide com o anel A-seco. A comparação dos dados de $\mathrm{RMN}{ }^{1} \mathrm{He}$ e ${ }^{13} \mathrm{C}$ com aqueles da literatura para a limonina permitiu confirmar a estrutura deste limonoide para a substância $\mathbf{1 2} .^{28}$ 
Segundo o sistema de classificação de Engler (1931) o gênero Euxylophora encontra-se classificado na subfamília Rutoideae, tribo Cusparieae. ${ }^{29}$ As classes de alcaloides mais abundantes em Rutaceae são aquelas derivadas do ácido antranílico. Entre estas, $2(1 H)$-quinolinonas, furoquinolinos, di-hidropiranoquinolinos, furo-4-quinolinonas, di-hidropirano-4-quinolinonas e pirano-2-quinolinonas seriam os que caracterizam a família como um todo. Outra classe que também caracteriza a família são as furanocumarinas. Em morfologia botânica considera-se como gênero típico aquele que possui o maior número de característica da família. Podem-se classificar estes alcaloides e as furanocumarinas como os grupos típicos de Rutaceae, pois são raros os gêneros em que eles ainda não foram citados. ${ }^{1,4}$ As furanocumarinas lineares isoladas nas folhas de E. paraensis são citadas em gêneros de todas as grandes subfamílias de Rutaceae, ${ }^{3,4}$ ou seja, confirmando que Euxylophora possui a maioria das características químicas das Rutaceae. Considerando o perfil químico de Euxylophora, este pode ser considerado um gênero típico de Rutaceae, pois como citado na introdução as cascas do caule e o cerne de sua única espécie possuem os alcaloides típicos da família. ${ }^{7-16}$ Estes trabalhos citam nos estudos com o cerne o isolamento de alcaloides simples do ácido antranílico (Figura 3) como 1-metil-4-(2,3-di-hidróxi-3-metilbutan-1-oxil)-2(1H)-quinolin-2-ona (13), furoquinolino (esquimmianina, 8), piranoquinolino ( $\mathrm{N}$-metilflindersina, 11), 1,2',2'-trimetil-3',4'-di-hidro,4'-hidróxi-pirano[3,2-c]<smiles>Cn1c(=O)cc(OCC(O)C(C)(C)O)c2ccccc21</smiles>

13

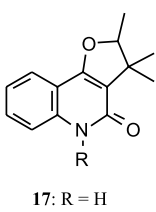

18: $\mathrm{R}=\mathrm{Me}$<smiles>Cn1c(=O)c2c(c3ccccc31)OC(C)(C)CC2O</smiles>

14

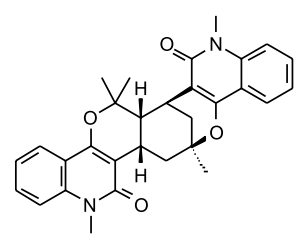

19

15
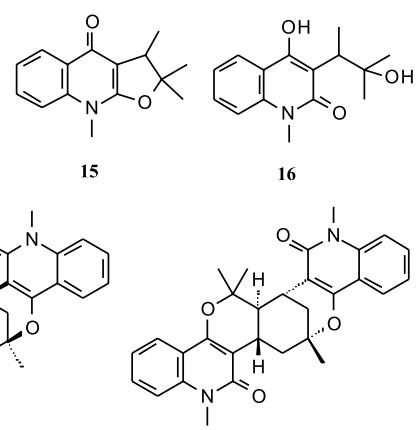

20<smiles>Cn1c(=O)c2c(c3ccccc31)OC(C)(C)CC21CCC(C)(O)CC12CCCC2</smiles>

21<smiles>Cn1c(=O)c2c(c3ccccc31)OC1c3c(c4ccccc4n(C)c3=O)OC(C)(C)C1CC(O)(O)C2</smiles>
24

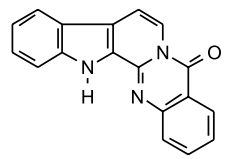

33: 2,3-diOMe

34: 2,3-diOMe, 14-M

35: 2,3,10-triOMe $2,3,10$-triOMe, $14-\mathrm{Me}$

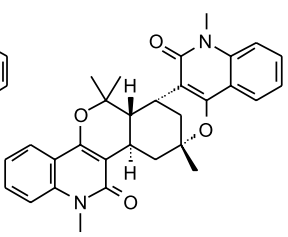

22

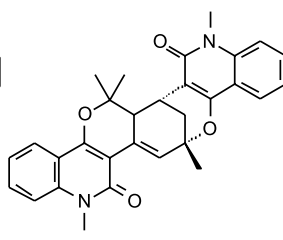

23
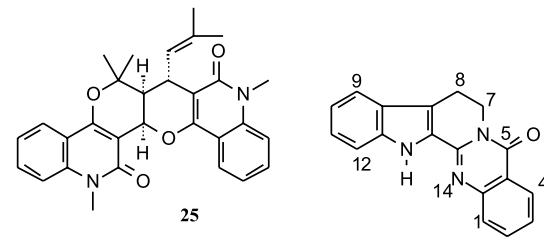

26: 1-OH

27: $2,3-\mathrm{OCH}_{2} \mathrm{O}$

28: $2-\mathrm{OH}, 3-\mathrm{OMe}$ 29: 2,3-diOMe 30: 2,3-diOMe, 14-Me

32: $2,3,10$-triOMe, $14-\mathrm{Me}$

Figura 3. Compostos citados na literatura de ocorrência em E. paraensis (os compostos 30, 32, 34 e 36 são iônicos) quinolin-2-ona (14), 1,2',2',3'-tetrametil-2',3'-di-hidrofuro[2,3-b] quinolin-4-ona (lemobilina, 15), 3-(1,2-dimetil-2-hidróxipropil)-4-hidróxi-1-metilquinolin-2(1H)-ona (16), 2',3',3'-trimetil-2',3'-di-hidrofuro[3,2-c]quinolin-2-ona (17), N-metil-2',3',3'-trimetil-2',3'di-hidrofuro[3,2-c] quinolin-2-ona (18). ${ }^{7-9,15}$ Nos compostos 15 e 16 o rearranjo de Claisen anormal parece ter ocorrido na sua formação, e 15 foi encontrado também em Flindersia ifflaiana, mostrando uma afinidade entre Euxylophora e este gênero, o qual pertence a outra subfamília, Flindersioideae..$^{30}$

Alguns gêneros de Rutaceae sintetizam alcaloides bis-2(1H)-quinolinona, estes em geral se formam por uma reação de cicloadição de Diels-Alder entre as unidades prenilas. Alguns destes dímeros foram isolados de Vepris louisii, Oricia renieri, E. paraensis e Ptelea trifoliata. ${ }^{30}$ Paraensidimerinas A (19), B (24), C (20), D (25), E (21), F (22), G (23) isolados do cerne de E. paraensis são exemplos da ocorrência da reação de Diels-Alder nestes dímeros. ${ }^{8,9,15,16}$ Vepris, Oricia e Ptelea são classificados em Toddalioideae.

O maior número de alcaloides isolados da casca do tronco de Euxylophora é derivado do ácido antranílico e triptofano, os $\beta$-indolo-quinazolinos: 1-hidróxi-7,8-di-hidro- $\beta$-indolo-quinazolino (26), euxylophoricinas A (29), B (33), C (27), D (31), E (35), F (28), euxylophorinas A (30), B (34), C (32), D (36) e paraensine (37). ${ }^{10-14}$ Os alcaloides $\beta$-indolo-quinazolinos segundo pesquisa recente foram isolados em três gêneros de Rutoideae, Zanthoxylum, Euodia e Euxylophora, e três de Toddalioideae, Vepris, Araliopsis e Hortia. ${ }^{30}$ Portanto, Euxylophora também mostra afinidade com gêneros da subfamília Toddalioideae, mais uma vez confirmando ser típico de Rutaceae. Dados micro e macromoleculares vêm sugerindo a união destas duas subfamílias, Rutoideae e Toddalioideae. ${ }^{31,32}$

Os flavonoides são geralmente encontrados em muitas, se não em todas, as plantas de Angiosperma, ${ }^{30}$ principalmente os glicosilados, portanto de pouco valor quimiossistemático na confirmação da classificação de um táxon. Contudo, os flavonoides de Rutaceae são tri-, tetra- e polioxigenados, uma exceção entre as demais angiospermas. As Rutaceae foram estudadas visando principalmente a busca de alcaloides, sendo em geral desprezadas as frações ricas em flavonoides. Recentemente estes compostos vêm sendo isolados em vários gêneros da tribo Cusparieae. Neoraputia e Conchocarpus são dois exemplos de gêneros ricos em flavonoides. ${ }^{30,33-35}$ A flavona 6 é pentaoxigenada, assim, também sugerindo afinidade de E. paraensis com os demais gêneros de Cusparieae.

\section{AGRADECIMENTOS}

À Capes, CNPq (INCT, 573742/2008-1) e FAPESP (INCT, 08/57859-5) pelas bolsas e apoios financeiros concedidos.

\section{REFERÊNCIAS}

1. Waterman, P. G.; Grundon, M. F.; Chemistry and Chemical Taxonomy of the Rutales, Academic Press: London, 1983.

2. Groppo, M.; Pirani, J. R.; Salatino, M. L. F.; Blanco, S. R.; Kallunki, J. A.; Am. J. Bot. 2008, 95, 985.

3. Silva, M. F. das G. F. da; Soares, M. S.; Fernandes, J. B.; Vieira, P. C.; The Alkaloids 2007, 64, 139.

4. Silva, M. F. das G. F. da; Gottlieb, O. R.; Ehrendorfer, F.; Plant Syst. Evol. 1988, 161, 97

5. Buchanan, B.; Gruissem, R.; Jones, R.; Biochemistry and molecular biology of plants, American Society of Plant Physiologists: New York, 2000.

6. http://www.ibama.gov.br/documentos/lista-de-especies-ameacadas-deextincao, acessada em Setembro 2012.

7. Jurd, L.; Wong, R. Y.; Aust. J. Chem. 1981, 34, 1625. 
8. Jurd, L.; Wong, R. Y.; Benson, M.; Aust. J. Chem. 1982, 35, 2505.

9. Danieli, B.; Manitto, P.; Ronchetti, F.; Russo, G.; Ferrari, G.; Experientia 1972, 28, 249.

10. Danieli, B.; Palmisano, G.; Rainoldi, G.; Russo, G.; Phytochemistry 1974, 13, 1603.

11. Danieli, B.; Farachi, G.; Palmisano, G.; Phytochemistry 1976, 15, 1095.

12. Canonica, L.; Danieli, B.; Manitto, P.; Russo, G.; Tetrahedron Lett. 1968, 47, 4865 .

13. Danieli, B.; Palmisano, G.; Russo, G.; Ferrari, G.; Phytochemistry 1973, $12,2521$.

14. Danieli, B.; Manitto, P.; Ronchetti, F.; Russo, G.; Ferrari, G.; Phytochemistry 1972, 11, 1833.

15. Jurd, L.; Benson, M.; Wong, R. Y.; Aust. J. Chem. 1983, 36, 759.

16. Jurd, L.; Benson, M.; J. Chem. Soc., Chem. Commun. 1983, $2,92$.

17. Müller, A. H.; Vieira, P. C.; Silva, M. F. das G. F. da; Fernandes, J. B.; Phytochemistry 1995, 40, 1797.

18. Stevenson, P. C.; Simmonds, M. S. J.; Yule, M. A.; Veitch, N. C.; Phytochemistry 2003, 63, 41.

19. Mikolajaczak, K. L.; Weisleder, D.; Parkanyl, L.; Clardy, J.; J. Nat. Prod. 1988, 51, 606.

20. Tesso, H.; Konig, W. A.; Kubeczka, M. B.; Glowniak, K.; Phytochemistry 2005, 66, 707.

21. Boyd, D. R.; Sharma, N. D.; Loke, P. L.; Malone, J. F.; Hamilton, T. G.; Chem. Commun. 2002, 3070.

22. Markham, K. R.; Chari, V. M.; Carbon-13 - NMR Spectroscopy of Flavonoids, Chapman and Hall Ltda: London, 1982.

23. Braca, A.; Politi, M.; Sanogo, R.; Sanou, H.; Morelli, I.; Pizza, C.; J. Agric. Food Chem. 2003, 51, 6689.

24. Chung, S.; Kim, Y.; Takaya, Y.; Terashima, K.; J. Agric. Food Chem. 2004, 52, 4664.

25. Jeong, S. H.; Huan, X. H.; Hong, S. S.; Hwang, J. S.; Hwang, J. H.; Lee, M. K.; Ro, J. S.; Hwang, B. Y.; Arch. Pharm. Res. 2006, 29, 1119.
26. Kim, J. S.; Kim, J. C.; Shim, S. H.; Lee, E. J.; Jin, W. J.; Son, K. H.; Kim, H. P.; Kang, S. S.; Chang, H. W.; Arch. Pharm. Res. 2006, 29, 617.

27. Kamperdick, C.; Van, N. H.; Sung, T. V.; Adam, G.; Phytochemistry 1999, 50, 177.

28. Ribeiro, T. A. N.; Ndiaye, E. A. da S.; Velozo, E. da S.; Vieira, P. C.; Ellena, J.; Júnior Sousa, P. T. de; J. Braz. Chem. Soc. 2005, 16, 1347.

29. Engler, A. Em Die Naturlichen Pflanzenfamilien; Engler, H. G. A.; Prantl, K., eds.; $2^{\text {nd }}$ ed., Wilhelm Engelmann: Leipzig, 1931, vol. 19a, p. 187-359.

30. Vieira, P. C.; Fernandes, J. B.; Oliva, G.; Silva, M. F. das G.F. da Em Química Medicinal: Métodos e fundamentos em planejamento de fármacos; Montanari, C. A., ed.; Editora da Universidade de São Paulo: São Paulo, 2011.

31. Severino, V. G. P.; Braga, P. A. C.; Silva, M. F. das G. F. da; Fernandes, J. B.; Vieira, P. C., Theodoro, J. E.; Ellena, J. A.; Phytochemistry 2012, $76,52$.

32. Braga, P. A C.; Severino, V. G. P.; Freitas, S. D. L. de; Silva, M. F. das G. F. da; Fernandes, J. B.; Vieira, P. C.; Pirani, J. R.; Groppo, M.; Biochem. Syst. Ecol. 2012, 43, 142.

33. Passador, E. A. P.; Silva, M. F. das G. F. da; Rodrigues Fo., E.; Fernandes, J. B.; Vieira, P. C.; Pirani, J. R.; Phytochemistry 1997, 45, 1533

34. Tomazela, D. M.; Pupo, M. T.; Passador, E. A. P.; Silva, M. F. das G. F. da; Vieira, P. C.; Fernandes, J. B.; Rodrigues Fo., E.; Oliva, G.; Pirani, J. R.; Phytochemistry 2000, 55, 643.

35. Moraes, V. R. de S.; Tomazela, D. M.; Ferracin, R. J.; Garcia, C. F.; Sannomiya, M.; Soriano, M. P. C.; Silva, M. F. das G. F. da; Vieira, P. C.; Fernandes, J. B.; Rodrigues Fo., E.; Magalhães, E. G.; Magalhães, A. F.; Pimenta, E. F.; Souza, D. H. F. de; Oliva, G.; J. Braz. Chem. Soc. 2003, 14, 380. 